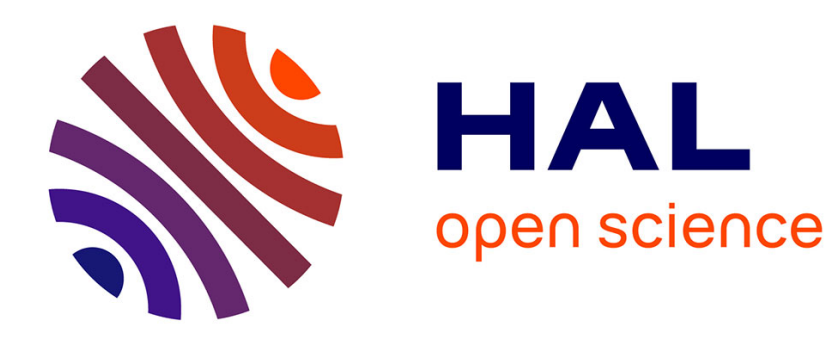

\title{
Variation of the exchange frequencies with density in bcc solid $3 \mathrm{He}$
}

\author{
M. Roger
}

\section{To cite this version:}

M. Roger. Variation of the exchange frequencies with density in bcc solid 3He. Journal de Physique Lettres, 1983, 44 (12), pp.481-490. 10.1051/jphyslet:019830044012048100 . jpa-00232220

\section{HAL Id: jpa-00232220 https://hal.science/jpa-00232220}

Submitted on 1 Jan 1983

HAL is a multi-disciplinary open access archive for the deposit and dissemination of scientific research documents, whether they are published or not. The documents may come from teaching and research institutions in France or abroad, or from public or private research centers.
L'archive ouverte pluridisciplinaire HAL, est destinée au dépôt et à la diffusion de documents scientifiques de niveau recherche, publiés ou non, émanant des établissements d'enseignement et de recherche français ou étrangers, des laboratoires publics ou privés. 
Classification

Physics Abstracts

$67.80-75.30 \mathrm{E}$

\title{
Variation of the exchange frequencies with density in bcc solid ${ }^{3} \mathrm{He}$
}

\author{
M. Roger \\ Institut Laue-Langevin, 156 X, 38042 Grenoble Cedex, France
}

(Reçu le 21 février 1983, révisé le 25 avril, accepté le 29 avril 1983)

\begin{abstract}
Résumé. - Nous discutons l'interprétation des mesures récentes de pression en champ magnétique, effectuées par Van Degrift et al. Contrairement aux auteurs, nous pensons que ces résultats ne sont pas en contradiction avec les mesures de susceptibilité et apportent des arguments supplémentaires en faveur du modèle d'échange multiple. Nous en déduisons les dérivées des fréquences d'échange à trois et quatre particules en fonction du volume et montrons qu'elles sont en accord avec les prédictions théoriques de récents calculs microscopiques.

Abstract. - We discuss the interpretation of recent pressure measurements in magnetic field performed by Van Degrift et al. In contrast with the authors, we think that these results are not in contradiction with the susceptibility measurements and give further arguments in favour of the multiple exchange model. We deduce the derivatives of the three and four particle exchange frequencies with respect to the volume and show that they agree with recent theoretical microscopic calculations.
\end{abstract}

\section{Introduction.}

The multiple exchange model [1, 2] has provided a quantitative explanation for most present experimental data on bcc solid ${ }^{3} \mathrm{He}$ at the melting molar volume. In this model cyclic three particle $\left(J_{t}\right)$ and four particle $\left(K_{\mathrm{p}}\right)$ exchange frequencies are of the same order of magnitude and other exchange processes (such as two particle transpositions) are neglected.

With the acquisition of new data at higher densities, important objections were recently raised against this model. Mamiya et al. [3] measured the ordering temperature $T_{c_{1}}$ and the coefficients of the high temperature specific heat series expansion $C_{\mathrm{v}}=0.25 R\left(\tilde{e}_{2} / T^{2}-\right.$ $\left.\tilde{e}_{3} / T^{3}+\tilde{e}_{4} / T^{4}\right)$; they found that $T_{c_{1}}, \tilde{e}_{2}^{1 / 2}, \tilde{e}_{3}^{1 / 3}, \tilde{e}_{4}^{1 / 4}$ all scale approximately on the same law $\sim v^{19 \pm 2}$. Devoret $e t$ al. [4] found that, in a wide volume range, the relaxation times $T_{1}, T_{2}$ roughly follow this same law. Hata et al. [5] measured the magnetization $M(T)$ versus temperature at several molar volumes $23<v<24.14 \mathrm{~cm}^{3} /$ mole and observed that the relation between the reduced magnetization $M / M_{\max }$ and the reduced temperature $T / T_{\mathrm{c}_{1}}$ is represented by a universal function. It was therefore argued that only one type of exchange should be significant. However, as shown in $[1,2]$, the magnetic properties of bcc ${ }^{3} \mathrm{He}$ at the melting molar volume cannot be interpreted with only one dominant exchange process (either two, three or four particle permutations). Another suggestion [6] was that three and four particle exchange frequencies have exactly the samc volume dependence, but this was at first sight difficult to understand on physical grounds.

New pressure measurements $P(T, v, H)$ in magnetic field, by Van Degrift et al. [7], recently provided accurate values of the derivative $\mathrm{d} \theta / \mathrm{d} v$ of the Curie Weiss constant $\theta$ in a small volume 
range $v=v_{0} \pm \Delta v$ with $v_{0} \simeq 24 \mathrm{~cm}^{3} /$ mole and $\Delta v / v<1.5 \%$. The authors fitted a power law : $\mathrm{d} \theta / \mathrm{d} v=A\left(v / v_{0}\right)^{\gamma}$ to their results and proposed an estimate of $\theta(v)$ by simple integration of this function. The values of $\theta$ thus obtained disagree strongly with all previous data deduced from susceptibility measurements. They suggest that this discrepancy might be due to some anomalous field dependence of $\theta$. However measurements in high magnetic field by Godfrin et al. exclude this hypothesis [8].

Another possible interpretation is the inadequacy of the power law to describe the functional dependence of $\theta$ on the molar volume.

The purpose of this letter is to show that there is no inconsistency with the susceptibility data if the results are interpreted in a more general context without assuming any particular functional dependence of $\theta$ on $v$ at $v_{0}=24 \mathrm{~cm}^{3}$. The fact that, in contrast to other quantities like $\tilde{e}_{2}, \theta$ does not accurately follow a power law favours a model with more than one exchange parameter. We analyse the results within the two parameters $\left(J_{t}, K_{\mathrm{p}}\right)$ multiple exchange Hamiltonian and deduce the Gruneisen parameters $\alpha^{\prime}=\mathrm{d} \ln J_{\mathrm{t}} / \mathrm{d} \ln v, \beta^{\prime}=\mathrm{d} \ln K_{\mathrm{p}} / \mathrm{d} \ln v$. These constants $\left(\alpha^{\prime}, \beta^{\prime}\right)$ are both close to 19 and differ only by a few unities. These results can be easily understood in terms of the underlying physics of the exchange processes used in recent theoretical calculations $[9,10]$; they explain reasonably well all present available experimental data.

\section{Consistent analysis of pressure and susceptibility data.}

Although a power law is generally used in the analysis of thermodynamic data for bcc ${ }^{3} \mathrm{He}$, it has no theoretical microscopic justification. It should only be considered as the first term of a series expansion. If the logarithm of some physical quantity $G$ varies smoothly with the molar volume one can use in a small volume range $v_{0} \pm \Delta v$ the following Taylor series expansion, limited to the first few terms :

$$
\ln G(v)=\ln G_{0}+g^{(1)} \frac{\Delta v}{v_{0}}+\frac{g^{(2)}}{2}\left(\frac{\Delta v}{v_{0}}\right)^{2}+\cdots+\frac{g^{(n)}}{n !}\left(\frac{\Delta v}{v_{0}}\right)^{n}+\cdots
$$

with $g^{(n)}=v_{0}^{n}\left[\mathrm{~d}^{n} \ln G / \mathrm{d} v^{n}\right]_{v=v_{0}} ; \Delta v=v-v_{0}$.

If $\ln G$ is sufficiently smooth and $\Delta v$ small (in bcc ${ }^{3} \mathrm{He}$ the volumes of physical interest do not exceed $v_{0} \pm \Delta v$ with $v_{0} \simeq 22 \mathrm{~cm}^{3} /$ mole and $\Delta v$ less than $12 \%$ ) a reasonable approximation can be obtained by retaining only the linear term in (1). This is strictly equivalent to assume either an exponential law $G=G_{0} \exp \left[g^{(1)}\left(\Delta v / v_{0}\right)\right]$ or a power law $G=G_{0}\left(v / v_{0}\right)^{g(1)}$.

Care should be taken when comparing data on the quantity $G(v)$ with data on its derivative $\mathrm{d} G / \mathrm{d} v$. We can write consistently with (1)

$$
\begin{aligned}
\ln \left(\frac{\mathrm{d} G}{\mathrm{~d} v}\right)=\ln \left(g^{(1)} G_{0} / v_{0}\right)+\left(g^{(1)}+g^{(2)} / g^{(1)}\right) & \frac{\Delta v}{v_{0}}+ \\
& +\frac{1}{2}\left[g^{(2)}+g^{(3)} / g^{(1)}-\left(g^{(2)} / g^{(1)}\right)^{2}\right]\left(\frac{\Delta v}{v_{0}}\right)^{2}+\cdots
\end{aligned}
$$

The first order term in (2) : $\gamma=g^{(1)}+g^{(2)} / g^{(1)}$ depends on both first and second order terms in (1). The procedure used in [7] neglects $g^{(2)} / g^{(1)}$ compared to $g^{(1)}$. If both terms are comparable, it becomes inadequate.

Through the fit

$$
\frac{\mathrm{d} \theta}{\mathrm{d} v}=\frac{27.8}{v_{0}} \mathrm{mK}\left(\frac{v}{v_{0}}\right)^{20.7 \pm 2}
$$


in the small volume range $v=v_{0} \pm \Delta v$ with $v_{0}=24 \mathrm{~cm}^{3} / \mathrm{mole}$ and $\Delta v / v_{0}<1.5 \%$, Van Degrift et al. [7] determine in fact the linear term in (2) :

$$
\left\{\begin{array}{l}
g^{(1)}\left|\theta_{0}\right|=27.8 \\
\gamma=g^{(1)}+g^{(2)} / g^{(1)}=20.7 \pm 2
\end{array} \quad(G(v)=|\theta(v)|) .\right.
$$

We thus obtain two relations between three unknown parameters : the Curie Weiss constant $\theta_{0}$, the slope $g^{(1)}$ and the curvature $g^{(2)}$ of the function $\ln |\theta(v)|$ around $v_{0}=24 \mathrm{~cm}^{3} / \mathrm{mole}$. The hypothesis of zero curvature $: g^{(2)}=0$ (exponential law approximation) leads to $g^{(1)}=20.7$ and $\left|\theta_{0}\right|=1.34 \mathrm{mK}$. The power law approximation used by Van Degrift et al. [7] is similar, it corresponds to $g^{(2)} / g^{(1)}=-1, g^{(1)}=21.7$ and $\left|\theta_{0}\right|=1.28 \mathrm{mK}$. Both approximations (resp. curves $(b)$ and $(a)$ of Fig. 1) disagree strongly with the susceptibility data reported on figure 1. (We consider all data quoted in [7] and recent results obtained by T. Hata et al. [5].)

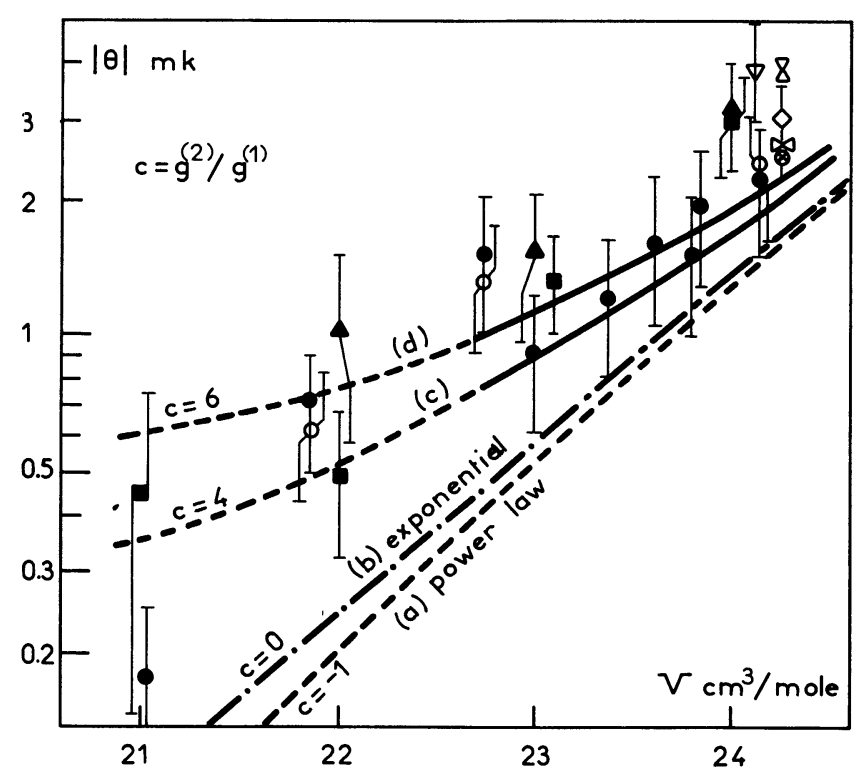

Fig. 1. - The values of the Curie Weiss constant $\theta$ obtained by susceptibility measurements are compared to different fits consistent with the pressure measurements of $\mathrm{d} \theta / \mathrm{d} v$ [7]. The experimental points are from : - Kirk et al. [12], $\diamond$ Sites et al. [13], $\nabla$ Johnson and Wheatley [14], $\Delta$ Bernat and Cohen [15], $\otimes$ Morii et al. [16], 8 Bakalyar et al. [17], $\bowtie$ Prewitt and Goodkind [18], Hata et al. (1981) [19], T. Hata et al. (1983) [5]. The four curves $(a)$ to $(d)$ are Taylor series expansions of $\ln |\theta|$ around $24 \mathrm{~cm}^{3}$, limited to second order and consistent with the data of Van Degrift et al. [7]. The curvature $c=g^{(2)} / g^{(1)}$ is increased from - 1 (power law fit $(a)$ ) to +6 (curve $d$ ). A reasonable agreement with the susceptibility data is obtained with $c \sim 4$ to 6 . At low molar volumes we expect third order terms to be significant and to lead eventually to an opposite curvature at high densities. For this reason our fits are plotted in dashed lines below $23 \mathrm{~cm}^{3} / \mathrm{mole}$.

Pressure and susceptibility data can however be reconciliated through the reasonable assumption that the curvature of $\ln |\theta(v)|$ at $v_{0} \simeq 24 \mathrm{~cm}^{3}$ is positive with $c=g^{(1)} / \mathrm{g}^{(2)}$ of the order of a few unities. The curves $(c)$ and $(d)$ of figure 1 obtained respectively with $\left(c=4, g^{(1)}=16.7\right.$, $\left.\left|\theta_{0}\right|=1.66\right)$ and $\left(c=6, g^{(1)}=14.7,\left|\theta_{0}\right|=1.89\right)$ are in reasonable agreement with all susceptibility data. 
We must emphasize that our series expansion limited to the second order might fail if we attempt to apply it too far from $v_{0}$. At $v \lesssim 23 \mathrm{~cm}^{3} / \mathrm{mole}$, higher order term are certainly significant. Pressure measurements at lower molar volumes could allow one to take the third order term $g^{(3)}$ (see relations 1,2$)$ into account and lead to a consistent fit in the whole volume range of interest for bcc ${ }^{3} \mathrm{He}$.

Further experimental data are needed to test our interpretation, in particular an accurate determination of $\theta$ by susceptibility measurements has to be performed at least at one particular molar volume. The large errors bars on present susceptibility data are mainly due to the fact that at $T \lesssim 2$ to $3|\theta|$ the susceptibility deviates strongly from the Curie Weiss law [17-20]. Experimentalists generally measure the magnetization at low field, deduce the susceptibility $\chi$ and fit the high temperature series expansion limited to the three first terms

$$
\chi^{-1}(T)=N^{-1}\left(\gamma \frac{\hbar}{2}\right)^{-2}\left(T-\theta+\frac{B}{T} \cdots\right) .
$$

As the number $N$ of the ${ }^{3} \mathrm{He}$ atoms in the cell is generally unknown, the three parameters $N$, $\theta, B$ are simultaneously determined in the fit. They are strongly correlated. For example an overestimate of $|B|$ leads to an overestimate of $|\theta|$. We point out that a way to eliminate one unknown parameter : $N$ in relation 5 is to use the multiple echo method of Bernier Delrieu [20]. This method indeed directly measures the magnetization density; thus the fit of the susceptibility only depends on two parameters $\theta$ and $B$, this method is more accurate than conventional measurements.

\section{Comparison of pressure measurements at zero field with specific heat data.}

The same problem arises when comparing the specific heat data : $C_{\mathrm{v}}=R \tilde{e}_{2} / 4 T^{2}$, giving $\tilde{e}_{2}(v)$, with the pressure measurements in zero field $P(T, v, H=0)=\left(\mathrm{d} \tilde{e}_{2} / \mathrm{d} v\right) / 8 T$, giving the derivative $\mathrm{d} \tilde{e}_{2} / \mathrm{d} v$. From the pressure measurements of Panczyck and Adams [21]:

$$
\mathrm{d} \tilde{e}_{2} / \mathrm{d} v=\frac{186.3}{v_{0}} \mathrm{mK}^{2}\left(\frac{v}{v_{0}}\right)^{35.26},
$$

we deduce at $v_{0}=24 \mathrm{~cm}^{3} /$ mole

$$
\left\{\begin{array}{l}
g^{(1)} \tilde{e}_{2}^{0}=186.3 \text { and } \\
\gamma=g^{(1)}+g^{(2)} / g^{(1)}=35.26
\end{array} \quad(G(v)=\tilde{e}(v) \text { in rel. }(1),(2)) .\right.
$$

On figure 2, we have reported the specific heat data for $23<v<24.5 \mathrm{~cm}^{3} / \mathrm{mole}$ from Castle and Adams [22], Greywall [23] and Hebral et al. [24]. We compare the exponential fit (curve (a)) : $g^{(2)}=0, g^{(1)}=35.26, \tilde{e}_{2}^{0}=5.28$ with two other fits :

$$
\begin{aligned}
& \text { (b) }: g^{(1)} / g^{(2)}=-2, g^{(1)}=37.26, \tilde{e}_{2}^{0}=5.00 \\
& (c): g^{(1)} / g^{(2)}=+2, g^{(1)}=33.26, \tilde{e}_{2}^{0}=5.60
\end{aligned}
$$

In this case the exponential fit $(a)$ gives the best agreement with the specific heat data. In contrast to $\theta, \tilde{e}_{2}$ can be reasonably approximated by an exponential law (or a power law) in the volume range of physical interest for bcc ${ }^{3} \mathrm{He}$. The functional dependences of $e_{2}^{1 / 2}$ and $\theta$ on $v$ around $v_{0}=24 \mathrm{~cm}^{3} /$ mole are thus different; this is an important argument in favour of a model with more than one exchange parameter. 


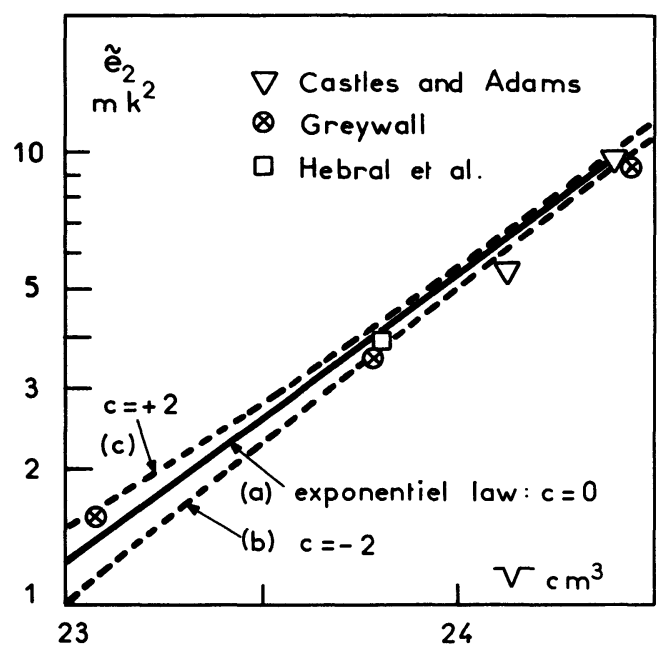

Fig. 2. - The values of $\tilde{e}_{2}$ deduced from the specific heat measurements of references 22-24 are compared to different Taylor series fits consistent with the pressure measurements [21]. In contrast to figure 1 , the best fit $(a)$ is the exponential law with zero curvature $c=0$.

\section{Analysis of the experimental data within the multiple exchange model.}

In this paragraph, we consider at $v_{0}=24 \mathrm{~cm}^{3} /$ mole the following experimental values which are determined unambiguously :

$$
\tilde{e}_{2} \simeq 5.3 \pm 0.15 \mathrm{mK}^{2},
$$

(5a) from pressure and specific heat measurements [21-24],

$v_{0} \frac{\mathrm{d} \tilde{e}_{2}}{\mathrm{~d} v}=186.3 \mathrm{mK}^{2}$

$v_{0}^{2} \frac{\mathrm{d}^{2} \tilde{e}_{2}}{\mathrm{~d} v^{2}}=35.26 \times 186.3 \mathrm{mK}^{2}$

$v_{0} \frac{\mathrm{d} \theta}{\mathrm{d} v}=27.8 \mathrm{mK}$

$v_{0}^{2} \frac{\mathrm{d}^{2} \theta}{\mathrm{d} v^{2}}=20.7 \times 27.8 \mathrm{mK}$

(We do not use experimental values of $\theta$ which still lead to controversies.)

We analyse the variations of the three particle $\left(J_{1}\right)$ and four particle $\left(K_{\mathrm{p}}\right)$ exchange frequencies with the density within the multiple exchange model.

We denote the logarithmic derivatives of the exchange frequencies by :

$$
\begin{aligned}
\alpha^{\prime} & =\mathrm{d} \ln J_{\mathrm{t}} / \mathrm{d} \ln v ; & & \beta^{\prime}=\mathrm{d} \ln K_{\mathrm{p}} / \mathrm{d} \ln v \\
\alpha^{\prime \prime} & =v_{0}^{2} \mathrm{~d}^{2} \ln J_{\mathrm{t}} / \mathrm{d} v^{2} ; & & \beta^{\prime \prime}=v_{0}^{2} \mathrm{~d}^{2} \ln K_{\mathrm{p}} / \mathrm{d} v^{2}
\end{aligned}
$$

(all derivatives are taken at $v_{0}=24 \mathrm{~cm}^{3} /$ mole), and the ratio between $J_{\mathfrak{l}}, K_{\mathrm{p}}$ by $\kappa=J_{\mathrm{t}} / K_{\mathrm{p}}$. 
In the two parameter multiple exchange model $\tilde{e}_{2}$ and $\theta$ are expressed in terms of the exchange parameters $K_{\mathrm{p}}, \kappa$ by $[1,2]$ :

$$
\begin{aligned}
\theta & =18(-2 \kappa+1) K_{\mathrm{p}} \\
\tilde{e}_{2} & =3\left(192 \kappa^{2}-168 \kappa+51\right) K_{\mathrm{p}}^{2}
\end{aligned}
$$

taking the derivatives with respect to the molar volume we obtain :

$$
\begin{aligned}
v_{0} \frac{\partial \theta}{\partial v} & =18\left(-2 \kappa \alpha^{\prime}+\beta^{\prime}\right) K_{\mathrm{p}}, \\
v_{0} \frac{\partial \tilde{e}_{2}}{\partial v} & =3\left[384 \alpha^{\prime} \kappa^{2}-168\left(\alpha^{\prime}+\beta^{\prime}\right) \kappa+102 \beta^{\prime}\right] K_{\mathrm{p}}^{2}, \\
v_{0}^{2} \frac{\partial^{2} \theta}{\partial v^{2}} & =18\left[-2\left(\alpha^{\prime \prime}+\alpha^{\prime 2}\right) \kappa+\beta^{\prime \prime}+\beta^{\prime 2}\right] K_{\mathrm{p}}^{2}, \\
v_{0}^{2} \frac{\partial^{2} \tilde{e}_{2}}{\partial v^{2}} & =3\left\{384\left[\alpha^{\prime \prime}+2 \alpha^{\prime 2}\right] \kappa^{2}-168\left[\alpha^{\prime \prime}+\beta^{\prime \prime}+\left(\alpha^{\prime}+\beta^{\prime}\right)^{2}\right] \kappa+102\left[\beta^{\prime \prime}+2 \beta^{\prime 2}\right]\right\} .
\end{aligned}
$$

Using the five experimental data (5a-e) we obtain five relations (6b-f) between six unknown parameters $K_{\mathrm{p}}, \kappa, \alpha^{\prime}, \beta^{\prime}, \alpha^{\prime \prime}, \beta^{\prime \prime}$. We can deduce $\alpha^{\prime}, \beta^{\prime}, \alpha^{\prime \prime}, \beta^{\prime \prime}$ and $\theta$ in terms of one variable $: \kappa$. The results are shown on figure 3 .

At melting densities the experimental data on $\tilde{e}_{2}$ and on the mean spin wave velocity restrict the parameters $\left(J_{t}, K_{\mathrm{p}}\right)$ to a small domain, as shown on figure 24 in reference 2 . In this domain $0.32 \lesssim \kappa \lesssim 0.38$. As we do not expect a large variation of $\kappa$ between 24.2 and $24 \mathrm{~cm}^{3} / \mathrm{mole}$ (see next paragraph) we have restricted figure 3 to $0.3<\kappa<0.4$. In this range $\beta^{\prime}$ is of the order of 17 to 20 and $\alpha^{\prime}$ differs from $\beta^{\prime}$ only by a few unities. This result is clearly understood within recent microscopic exchange calculations $[9,10,11,26]$.

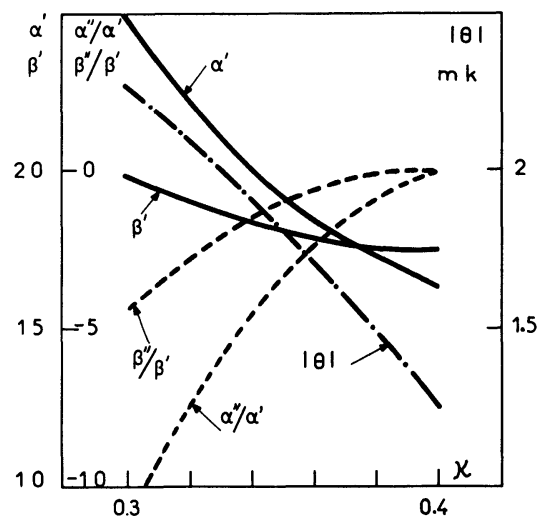

Fig. 3. - The logarithmic derivatives $\alpha^{\prime}=\mathrm{d} \ln J_{\mathrm{t}} / \mathrm{d} \ln v, \quad \beta^{\prime}=\mathrm{d} \ln K_{\mathrm{p}} / \mathrm{d} \ln v, \quad \beta^{\prime \prime}=v_{0}^{2} \mathrm{~d}^{2} \ln J_{\mathrm{t}} / \mathrm{d} v^{2}$, $\beta^{\prime \prime}=v_{0}^{2} \mathrm{~d}^{2} \ln K_{\mathrm{p}} / \mathrm{d} v^{2}$ at $v_{0}=24 \mathrm{~cm}^{3}$ and the Curie Weiss constant $\theta$ are deduced in terms of $\kappa=J_{\mathrm{t}} / K_{\mathrm{p}}$ from precise experimental values of $\tilde{e}_{2}, \mathrm{~d} \tilde{e}_{2} / \mathrm{d} v, \mathrm{~d}^{2} \tilde{e}_{2} / \mathrm{d} v^{2}, \mathrm{~d} \theta / \mathrm{d} v$ and $\mathrm{d}^{2} \theta / \mathrm{d} v^{2}$.

\section{Comparison with the predictions of recent microscopic calculations.}

The functional form for the volume dependence of the exchange parameters in the high density limit has been determined simultaneously by Avilov and Iordansky [11] and Roger $[9,10]$. 
At high densities, the potential energy (mainly the repulsive part of the Lennard Jones potential $\left.\sim 4 \varepsilon(\sigma / r)^{12}\right)$ becomes more important than the kinetic energy of localization of the atoms and a multidimensional WKB expansion can be used. In reduced units $\rho=\mathbf{r} / a$ ( $a$ interatomic distance), the Schrödinger equation is written :

$$
-\frac{\hbar^{2}}{2 m a^{2}} \Delta \psi(\rho)+4 \varepsilon\left(\frac{\sigma}{a}\right)^{12} V(\rho) \cdot \psi(\rho)=E \psi(\rho),
$$

with $V(\boldsymbol{\rho})=\sum_{1<j}\left|\rho_{i j}\right|^{-12}$.

The significant parameter $g$ for the WKB series expansion is the square root of the ratio between the kinetic and potential energy terms i.e. $g \sim \hbar\left(\frac{a}{\sigma}\right)^{5}$. At lowest order in $g$, the exchange frequencies $J_{\mathrm{p}}$ are proportional to $\exp \left(-\frac{S_{\mathrm{c}_{1}}}{g}\right)$ where $S_{\mathrm{c}_{1}}=\int_{\rho_{1}}^{\rho_{2}} \sqrt{8 m \varepsilon(V[\rho(l)]-E)} \mathrm{d} l$ is the classical action for a point mass $m$ moving in the $3 \mathrm{~N}$ dimensional configuration space representing the coordinates $\rho$ of $N$ distinguishable particles in a potential minus $V(\boldsymbol{\rho})$ [25]. The extremities $\boldsymbol{\rho}_{1}, \boldsymbol{\rho}_{2}$ of the exchange path are two equilibrium configurations deduced through the permutation $P$ of $n_{\mathrm{e}}$ particles. As $g$ varies as $v^{5 / 3}$ we obtain at lowest order in $g$ the following functional dependence of $J_{\mathrm{p}}$ on $v$ :

$$
J_{\mathrm{p}}=A_{\mathrm{p}} \exp \left[-S_{\mathrm{p}}\left(\frac{v}{v_{0}}\right)^{-5 / 3}\right]
$$

This calculation strictly applies for three dimensional hcp ${ }^{3} \mathrm{He}$ or 2 dimensional triangular lattice when the interatomic spacing $a$ is comparable to $\sigma$. It certainly fails for bcc ${ }^{3} \mathrm{He}$ at densities near melting.

For the lower densities of bcc ${ }^{3} \mathrm{He}$ the kinetic energy of localization for the particles and the attractive part of the Lennard Jones potential have to be taken carefully into account. Several approximations have been worked out. Delrieu and Sullivan [26] proposed a rough elastic model where an effective potential barrier for tunnelling is estimated from the experimental pressure and compressibility of bcc ${ }^{3} \mathrm{He}$. The same idea was formulated more recently by Avilov and Iordansky [11] with a more careful optimization of the tunnelling path; they use the WKB expansion described above with an effective potential $V(\mathbf{r})$ which is estimated from the experimental results on the elastic properties of bcc ${ }^{3} \mathrm{He}$.

A remarkable experimental fact is that all quantities related to the elastic properties of solid ${ }^{3} \mathrm{He}$ (or ${ }^{4} \mathrm{He}$ ) seem to scale according to universal power laws in terms of the molar volume $v$. For example pressure and compressibility vary as $v^{-5}$, the Debye frequency varies as $v^{-7 / 3} \ldots$ $[10,11,17]$. Using these results, the effective potentials $V(\mathbf{r})$ estimated by $[11,26]$ vary as $v^{-4}$. Hence the classical action $S_{\mathrm{c}_{1}} \sim \int_{\mathrm{r}_{1}}^{\mathrm{r}_{2}} \sqrt{V[\mathrm{r}(l)]} \mathrm{d} l$ varies as $v^{-2} \cdot v^{1 / 3}=v^{-5 / 3}$, and we are lead to the same functional dependence as expressed in relation 7 for the exchange frequencies $J_{\mathbf{p}}$.

The preexponential factor $A_{\mathrm{p}}$ in relation 7 is difficult to calculate exactly. To some numerical constant of the order of a few unities, it is roughly equal to the Debye temperature $\theta_{\mathbf{D}}$. As a rough evaluation, Avilov and Iordansky [11] proposed :

$$
A_{\mathrm{p}}=Z n_{\mathrm{e}}\left(\frac{m a^{2}}{\hbar^{2}} \cdot \frac{\theta_{\mathrm{D}}}{\pi}\right)^{1 / 2} \cdot \theta_{\mathrm{D}}
$$

( $Z$ is the number of equivalent tunnelling paths and $n_{\mathrm{e}}$ the number of exchanging particles). 
We must emphasize that this functional form [7, 8] represents essentially the lowest order terms of a high density series expansion in powers of $a / \sigma$. For the densities of bcc ${ }^{3} \mathrm{He}$ the kinetic energy of the particles is of the same order of magnitude as the potential energy and the application of this formula based on the WKB approximation might be suspect. However it is interesting to compare its predictions for the Grüneisen constants $\alpha^{\prime}, \beta^{\prime}$ to the value deduced from the experiments (cf. § 4).

The factors $A_{\mathrm{p}}$ corresponding to different kinds of exchange (three, four particle exchanges) only differ by a factor close to one and the hierarchy between the exchange frequencies is essentially governed by the exponent $S_{\mathrm{p}}$ in the exponential. If we compare three and four particle exchange, we can write from [7]

$$
\ln \left(J_{\mathrm{t}} / K_{\mathrm{p}}\right)=\ln \kappa \simeq\left(S_{4}-S_{3}\right)\left(\frac{v}{v_{0}}\right)^{-5 / 3}
$$

where $S_{3}$ and $S_{4}$ denote the constant $S_{\mathrm{p}}$ for three and four particle exchange respectively. We deduce

$$
\alpha^{\prime}-\beta^{\prime}=[\partial \ln \kappa / \partial \ln v]_{v=v_{0}} \simeq \frac{5}{3}\left(S_{3}-S_{4}\right)
$$

or $\alpha^{\prime}-\beta^{\prime} \simeq-\frac{5}{3} \ln \kappa$.

If we now consider the results of the preceding paragraph (Fig. 3) we find that this relation (9) is approximately verified for $\kappa \simeq 0.34$, corresponding to $\theta \simeq-1.9 \mathrm{mK}$, in good agreement with the fit $(d)$ of figure 1 . We obtain $J_{\mathrm{t}} \simeq 0.11 \mathrm{mK}, K_{\mathrm{p}} \simeq-0.33 \mathrm{mK}, \alpha^{\prime} \simeq 20, \beta^{\prime} \simeq 18.3$, $\alpha^{\prime \prime} / \alpha^{\prime} \simeq-1.5$ and $\beta^{\prime \prime} / \beta^{\prime} \simeq-4.5$.

It is important to check if these values are consistent with relation 7 , keeping in mind that it gives only the order of magnitude of $J_{\mathrm{p}}$. We take the estimate of $A_{\mathrm{p}}$ proposed by Avilov and Iordansky (rel. 8) with the experimental value $\theta_{\mathrm{D}} \simeq 18 \mathrm{~K}$ at $24 \mathrm{~cm}^{3} / \mathrm{mole}$. Putting $K_{\mathrm{p}} \simeq 0.33 \mathrm{mK}$ in relations 7,8 we find $S_{4}=\ln \left(A_{\mathrm{p}} / K_{\mathrm{p}}\right) \simeq 13.25$. As $\theta_{\mathrm{D}}$ varies experimentally as $v^{-7 / 3}, A_{\mathrm{p}}$ varies as $v^{-19 / 6}$ (cf. [11]) and we have $\beta^{\prime}=\mathrm{d} \ln K_{\mathrm{p}} / \mathrm{d} \ln v=\frac{5}{3} S_{4}-\frac{19}{6} \simeq 18.9$ in reasonable agreement with the preceding value 18.3.

The Grüneisen parameters $\alpha^{\prime}, \beta^{\prime}$ deduced from the experiments $(\S 4)$ are thus in good agreement with the values deduced from relations 7,8 , indicating that the functional form (7) remains roughly valid for bcc ${ }^{3} \mathrm{He}$.

For the second derivatives we predict from $[7,8] \beta^{\prime \prime}=\left(-\frac{8}{3}\right) \cdot\left(\frac{5}{3}\right) S_{4}+\frac{19}{6}=-55.7$ leading to $\beta^{\prime \prime} \mid \beta^{\prime}=-2.9$; we obtain roughly the same value for $\alpha^{\prime \prime} / \alpha^{\prime}$. The values $\alpha^{\prime \prime} / \alpha^{\prime} \simeq-1.5$ and $\beta^{\prime \prime} \mid \beta^{\prime} \simeq-4.5$ deduced from figure 3 at $\kappa=0.34$ disagree with the predictions of $[7,8]$. This means that corrections to $[7,8]$ due to quantum fluctuations around the classical path are significant. However the disagreement for each value is less than $50 \%$ and the sign is respected; for this reason we think that those corrections do not change drastically the functional form (7).

The value $\theta=-1.28$ proposed by Van Degrift et al. leads to $\kappa \sim 0.4, \alpha^{\prime}-\beta^{\prime}<0, \alpha^{\prime \prime} \sim \beta^{\prime \prime} \sim 0$, in complete contradiction with the relations 7-9.

We note that the laws $(7,8)$ gives a negative curvature $\mathrm{d}^{2} \ln J_{\mathrm{p}} / \mathrm{d} v^{2}$. At high densities we expect that the exchange constants follow relation 7 exactly. In this limit, the Curie Weiss constant will be proportional to the dominant exchange $K_{\mathrm{p}}$ and the curvature of the function $\ln |\theta(v)|$ will be negative. From the experimental data we deduced (cf. $\S 2$ ) a positive curvature at $v_{0}=24 \mathrm{~cm}^{3} /$ mole. Consequently the curvature of $\ln |\theta|$ must change of sign at low $v$. It would be interesting to determine if this change of sign occurs between 19.8 and $24 \mathrm{~cm}^{3} / \mathrm{mole}$ or below the instability to the hcp phase. 
We have analysed.all data within a two parameter Hamiltonian (three + four spin exchange : $J_{t}, K_{\mathrm{p}}$ ) neglecting completely pair exchange $J_{\mathrm{NN}}$. Pair exchange $J_{\mathrm{NN}}$ might happen to be significant. The calculation of Avilov and Iordansky [11] leads in fact to $\left|J_{N N}\right|>\left|J_{1}\right|$. However the classical tunnelling path is determined with only a small number of moving particles (exchanging atoms plus one to four neighbours). We think that especially at high densities a reasonable comparison between different exchange frequencies cannot be obtained with less than 15 to 20 moving particles (exchanging particles plus a reasonable number of moving neighbours). We have performed the high density quasi classical approximation with a larger number of variables ( $\sim 15$ moving neighbours) and found the hierarchy $\left|J_{N N}\right| \lesssim\left|J_{t}\right|<\left|K_{\mathrm{p}}\right|$ in agreement with our two parameter model. More accurate experimental data are needed to determine if $J_{\mathrm{NN}}$ is really significant.

\section{Interpretation of the other experimental results.}

With the values $\alpha^{\prime} \simeq 20$ and $\beta^{\prime} \simeq 18.3$ deduced in the preceding paragraph, we easily explain that the logarithmic derivatives of most physical quantities which have the same dimension as $J_{t}, K_{\mathrm{p}}$ turn out to be around 18 to 20 . Let us take for example the ordering temperature $T_{\mathrm{c}_{1}}$. The molecular field estimate is $[1,2] T_{\mathrm{c}_{1}}=-\left(2 J_{\mathrm{t}}+\frac{3}{2} K_{\mathrm{p}}\right) / \ln 2$. We deduce

$$
\mathrm{d} \ln T_{\mathrm{c}_{1}} / \mathrm{d} \ln v=\left(2 \alpha^{\prime} \kappa+3 \frac{\beta^{\prime}}{2}\right) /\left(2 \kappa+\frac{3}{2}\right)=18.8
$$

in good agreement with the value $19.1 \pm 0.2$ proposed in [3].

A quasi universal behaviour of the susceptibility in the small volume range $23<v<$ $24.2 \mathrm{~cm}^{3} /$ mole [5] can also be easily understood within this model. To test our interpretation, it would be interesting to perform new experiments which could measure separately the exchange constants $J_{1}, K_{\mathrm{p}}$ or directly their ratio $\kappa$ at various densities. A neutron scattering experiment in the paramagnetic phase, around to $10 \mathrm{mK}$ has been recently suggested to determine separately the different exchange constants [28]. The ratio $\kappa$ could also be simply obtained from the magnetization of the high field phase at low $T$, near the transition with the low field phase. This magnetization is $\sqrt{\kappa} \cdot M_{\mathrm{s}}\left(M_{\mathrm{s}}\right.$ : saturation magnetization). It has only been measured at melting. From the preceding sections we predict a slow variation of $\kappa$ with $\partial \ln \kappa / \partial \ln v \simeq 1$. This slow variation of $\kappa$ implies that the changes of the magnetic phase diagram that we suggested [2] for low molar volumes can be suppressed by the structural phase transition. For example the change of the low field ordered phase uudd to ssqaf [2] should happen for $\kappa \leqslant 0.25$. Using the preceding relation for $\kappa$, this would correspond to $v \simeq 20 \mathrm{~cm}^{3}$ just near the instability to the hcp phase.

\section{Conclusion.}

All present data on the density dependence of the experimental data in bcc ${ }^{3} \mathrm{He}$ are understood within the two parameter multiple exchange model. The Grüneisen constants $\alpha^{\prime}=\mathrm{d} \ln J_{\mathrm{t}} / \mathrm{d} \ln v$ and $\beta^{\prime}=\mathrm{d} \ln \kappa_{\mathrm{p}} / \mathrm{d} \ln v$ are close in value to $18 \sim 20$ and differ only by a few unities : $\alpha^{\prime}-\beta^{\prime} \sim$ +1 to +2 . This result is in reasonable agreement with the functional form $J_{p}$

$$
J_{\mathrm{p}} \sim A_{\mathrm{p}} \exp \left[-S_{\mathrm{p}}\left(\frac{v}{v_{0}}\right)^{-5 / 3}\right]
$$

extrapolated from high density WKB series expansions [9-11].

This model provides a coherent explanation to all present data in bcc solid ${ }^{3} \mathrm{He}$. Further 
experimental investigations, in particular better direct measurements of the Curie Weiss constant $\theta$ are needed to test our interpretation.

The two parameter multiple exchange Hamiltonian is the simplest model which gives a satisfying agreement with all present data. However the physics of ${ }^{3} \mathrm{He}$ might be more complicate, in particular pair exchange that we have completely neglected might be significant. In microscopic calculations we have also neglected vacancies. If zero point vacancies are present, with a significant concentration, they might play an important role ; in particular three and four spin exchange frequencies would be thus strongly correlated.

\section{Acknowledgments.}

It is a pleasure to thank H. Godfrin, B. Castaing, J. M. Delrieu and N. Sullivan for stimulating discussions.

\section{References}

[1] Roger, M., Delrieu, J. M. and Hetherington, J. H., J. Physique Colloq. 41 (1980) C7-241.

[2] Roger, M., Hetherington, J. H. and Delrieu, J. M., Rev. Mod. Phys. 55 (1983) 1.

[3] Mamiya, T., Sawada, A., Fukuyama, H., Hiro, Y. and Masuda, Y., Phys. Rev. Lett. 471304.

[4] Devoret, M., Greenberg, A. S., Esteve, D., Sullivan, N. S. and Chapellier, M., J. Low Temp. Phys. 48 (1982) 495.

[5] Hata, T., Yamasaki, S., Taneda, M., Kodama, T., Shigi, T., Proc. of ICM'82 Conference (Kyoto, Sept. 1982), to appear in J. Magn. Magn. Mat.

[6] IChiKaWA, K., Proc. of ICM'82 Conference (Kyoto, Sept. 1982), to appear in J. Magn. Magn. Mat.

[7] Van Degrift, C. T., Bowers, W. J., PiPes, P. B. and McQueEney, D. F., Phys. Rev. Lett. 49 (1982) 149.

[8] Godfrin, H., Frossati, G., Greenberg, A. S., Hebral, B., Thoulouze, D., to appear in J. Phys. Lett.

[9] Roger, M., Proc. of ICM' 82 Conference (Kyoto, Sept. 1982) to appear in J. Magn. Magn. Mat.

[10] ROGER, M., to appear.

[11] Avilov, V. V. and Iordansky, S. V., J. Low Temp. Phys. 48241.

[12] Kirk, W. P., Osgood, E. B. and Garber, M., Phys. Rev. Lett. 23 (1969) 833.

[13] Sites, J. R., Osheroff, D. D., Richardson, R. C. and Lee, D. M., Phys. Rev. Lett. 23 (1969) 836.

[14] Johnson, R. T. and Wheatley, J. C., Phys. Rev. A 1 (1970) 1836.

[15] Bernat, T. P. and Cohen, H. D., Phys. Rev. A 7 (1973) 1709.

[16] Morit, Y., Ichikawa, K., Hata, T., Kanamori, C., Hokamoto, H., Kodama, T. and Shigi, T. in Physics of Ultra-low Temperatures, Sugawara, T. et al. Eds. (Physical Society of Japan, Tokyo) 1978, p. 196.

[17] Bakalyar, D. M., Britton, C. V., Adams, E. D. and Huang, Y. C., Phys. Lett. 64A (1977) 208.

[18] Prewitt, T. C. and Goodkind, J. M., Phys. Rev. Lett. 44 (1980) 1699.

[19] Hata, T., Yamasaki, S., Taneda, Y. and Shigi, T., Physica 107B (1981) 201.

[20] Bernier, M. and Delrieu, J. M., Phys. Lett. A 60 (1977) 156.

[21] Panczyck, M. F. and Adams, E. D., Phys. Rev. A 1 (1970) 1356.

[22] Castles, S. H. and Adams, E. D., Phys. Rev. Lett. 30 (1973) 1125.

[23] Greywall, D. S., Phys. Rev. B 15 (19\%7) 2604.

[24] Hebral, B., Frossati, G., Godfrin, H., Schumacher, G. and Thoulouze, D., J. Physique Lett. 40 (1979) L-41.

[25] For more details about the multidimensional WKB expansion, see for example GeRVAIS, J. L. and Sakita, B., Phys. Rev. D 163507.

[26] Delrieu, J. M. and Sullivan, N. S., Phys. Rev. B 23 (1981) 3197.

[27] Wilks, J., The properties of solid and liquid helium (Clarendon Press Oxford) 1967, p. 646.

[28] Roger, M. and Glyde, H., Phys. Lett. 89A (1982) 252. 